Bull. Austral. Math. Soc.

$51 \mathrm{~K} 05,51 \mathrm{M} 16$

VOL. 61 (2000) [109-119]

\title{
A MAXIMAL GROSS-STADJE NUMBER IN THE EUCLIDEAN PLANE
}

\section{F. Pillichshammer}

Let $X$ be a compact, connected Hausdorff space and $f$ a real valued, symmetric, continuous function on $X \times X$. Then the Gross-Stadje number $r(X, f)$ is the unique real number with the property that for each positive integer $n$ and for all (not necessarily distinct) $x_{1}, \ldots, x_{n}$ in $X$, there exists some $x$ in $X$ such that $\sum_{i=1}^{n} f\left(x_{i}, x\right)=n r(X, f)$. This paper solves the following open question in distance geometry: What is the least upper bound $g_{2}\left(\mathbf{R}^{2}\right)$ of $r\left(X, d^{2}\right)$, where $X$ ranges over all compact, connected subsets of the Euclidean plane with diameter one and where $d^{2}$ denotes the squared, Euclidean distance. We show: $g_{2}\left(\mathbf{R}^{2}\right)=3-\sqrt{6}$.

\section{INTRODUCTION}

Let $X$ be a compact, connected Hausdorff space and $f$ a real valued, symmetric, continuous function on $X \times X$. Then there is a unique real number $r(X, f)$ with the property that for each positive integer $n$ and for all (not necessarily distinct) $x_{1}, \ldots, x_{n}$ in $X$, there exists some $x$ in $X$ such that

$$
\frac{1}{n} \sum_{i=1}^{n} f\left(x_{i}, x\right)=r(X, f) .
$$

For the case when $f$ is a metric on $X \times X$ this result was proved by 0 . Gross [2] in 1964. The more general result stated above was proved by W. Stadje [3] (independendly from Gross) in 1981. The number $r(X, f)$ is called Gross-Stadje number and is associated with $X$ and the function $f$. If $f$ is a metric $d$, then $r(X, d)$ is also often called the rendezvous number of the metric space $(X, d)$. An excellent survey on this topic is given in [1].

In this paper we consider the case that $X$ is a subset of the Euclidean plane and $f$ is the squared, Euclidean distance $d^{2}$ (by $\|$.$\| we denote the Euclidean norm). In general$ the explicit calculation of the number $r(X, f)$ for a given compact, connected Hausdorff space $X$ and a real valued, symmetric, continuous function $f$ on $X \times X$ is rather difficult. It turns out that the calculation of $r\left(X, d^{2}\right)$ is much easier.

Received 13th May, 1999

This work was supported by the FWF Project P-12441 MAT. 1 would like to thank my doctoral thesis advisor Reinhard Wolf for valuable discussions and suggestions.

Copyright Clearance Centre, Inc. Serial-fee code: 0004-9727/00 \$A2.00+0.00. 
Theorem 1. (Wilson) Let $X$ be a compact, connected subset of $\mathbb{R}^{n}$. Let $B_{1}$ be a closed ball and $B_{2}$ an open ball such that $X$ is contained in $B_{1} \backslash B_{2}$ and the centre of each ball lies in the closed convex hull of the intersection of $X$ with the boundary of the other. Further, let $B_{1}$ have centre $u$ and radius $R$ and let $B_{2}$ have centre $v$ and radius $r$. Then

$$
r\left(X, d^{2}\right)=R^{2}+r^{2}-\|u-v\|^{2} .
$$

For a proof see [4]. The existence of the balls in Theorem 1 is also shown in Wilson's paper.

For example let $X$ be the Reuleaux triangle with diameter 1 . Choose $B_{1}$ as the convex hull of the circumscribed circle und $B_{2}$ as the interior of the convex hull of the inscribed circle. Then we get with the help of Wilson's Theorem $r\left(X, d^{2}\right)=(5-2 \sqrt{3}) / 3$. (Remember that $r(X, d)$ of the Reuleaux triangle is still unknown.) For more examples see $[1,4]$.

Define the number $m\left(X, d^{2}\right)$ as $r\left(X, d^{2}\right) / D\left(X, d^{2}\right)$, where $D\left(X, d^{2}\right)=\sup \left\{\|x-y\|^{2} \mid\right.$ $x, y \in X\}$ and $g_{2}\left(\mathbb{R}^{2}\right)$ as the supremum of the numbers $m\left(X, d^{2}\right)$ as $X$ ranges over all compact, connected subsets of $\mathbb{R}^{2}$. In [1] the authors ask for the value of $g_{2}\left(\mathbb{R}^{n}\right)$, which is defined analogously. All values $g_{2}\left(\mathbb{R}^{n}\right), n \geqslant 2$, are still unknown. The first information about the magnitude of $g_{2}\left(\mathbb{R}^{2}\right)$ is given in the following inequality: For all compact, connected metric spaces $(X, d)$ we have

$$
\frac{1}{4} \leqslant m\left(X, d^{2}\right)<1 .
$$

For a proof of this inequality see for example [1]. Wilson conjectured in [4] that $g_{2}\left(\mathbb{R}^{2}\right)=(3-\sqrt{11 / 3}) / 2$, which is the number $m\left(X, d^{2}\right)$ for two sides of a Reuleaux triangle. But we shall show that this value is a little bit too small.

\section{RESULTS}

The following Proposition leads to the calculation of $g_{2}\left(\mathbb{R}^{2}\right)$.

Proposition 1. Let $S_{1}$ be a circle with centre $u$ and radius $R$ and let $S_{2}$ be a circle with centre $v$ and radius $r, R \geqslant r \geqslant 0, R>0$ and $0 \leqslant\|u-v\| \leqslant R$. Let $X$ be a compact, connected subset of conv $S_{1} \backslash\left(\operatorname{conv} S_{2}\right)^{\circ}$ where $v$ is in $\operatorname{conv}\left(S_{1} \cap X\right)$ and $u$ is in $\operatorname{conv}\left(S_{2} \cap X\right)$. Then we have

$$
m\left(X, d^{2}\right) \leqslant 3-\sqrt{6} \approx 0.5505102 .
$$

Now we get 
Theorem 2. Define $g_{2}\left(\mathbb{R}^{2}\right)$ as in Section 1. Then we have

$$
g_{2}\left(\mathbf{R}^{2}\right)=3-\sqrt{6}
$$

REMARK 1. The value $3-\sqrt{6}$ is attained, for example for the following set: Let $S_{1}$ be a circle with centre $u$ and radius $R=1, S_{2}$ be a circle with centre $v$ and radius $r=\sqrt{3 /(4 \sqrt{6}-6)}$ and let $\|u-v\|=\sqrt{3 / 2}-1$. Let $\left\{x_{1}, x_{2}\right\}$ be the intersection of $S_{1}$ and $S_{2}$. Further let $x_{3}$ be the intersection point of $S_{1} \backslash$ conv $S_{2}$ and the line which is determined by $u$ and $v$ and let $x_{4}$ be the intersection point of $S_{2} \cap \operatorname{conv} S_{1}$ and the line which is determined by $u$ and $v$. Then define the set $A$ as follows: $A$ consists of the arc joining $x_{1}$ and $x_{2}$ in $S_{2} \cap$ conv $S_{1}$ and the line segment $x_{3} x_{4}$ (see Figure 1). Observe that $D\left(A, d^{2}\right)=\left\|x_{1}-x_{3}\right\|^{2}=(2 r)^{2}$.

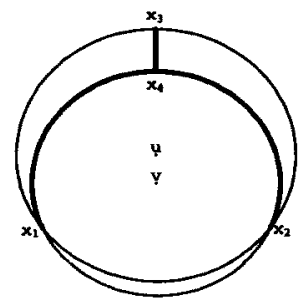

Figure 1: The set A.

\section{Proofs}

For the proof of Proposition 1 we need the following Lemmas:

LEMMA 1. Let $S$ be a circle with centre $u$ and radius $R$. Let $v$ be a point in conv $S$ and $g$ be the line with $v$ in $g$ and $g$ perpendicular to the line segment $u v$. Further let $h$ be an arbitrary line with $v$ in $h$. Then we have with $\left\{x_{1}, x_{2}\right\}=S \cap g$ and $\left\{y_{1}, y_{2}\right\}=S \cap h$

$$
\left\|x_{1}-x_{2}\right\| \leqslant\left\|y_{1}-y_{2}\right\| \text {. }
$$

The proof is straight forward.

Lemma 2. Let $S$ be a circle with centre $u$ and radius $R$. Let $x_{1}, x_{2}, x_{3}$ be points in $S$ with $u$ in $\operatorname{conv}\left\{x_{1}, x_{2}, x_{3}\right\}$. Then we have

$$
\max _{1 \leqslant i, j \leqslant 3}\left\|x_{i}-x_{j}\right\| \geqslant \sqrt{3} R .
$$

The proof is straight forward.

Lemma 3. Let $S$ be a circle and let $X$ be a subset of conv $S$ with $S \cap X$ is not empty. Let $v$ be a point in $\operatorname{conv}(S \cap X)$. Then there are points $x_{1}, x_{2}, x_{3}$ in $X \cap S$ with $v$ in conv $\left\{x_{1}, x_{2}, x_{3}\right\}$. 
The Lemma follows from Caratheodory's Theorem.

Lemma 4. Let $S_{1}$ be a circle with centre $u$ and radius $R$ and let $S_{2}$ be a circle with centre $v$ and radius $r, R \geqslant r \geqslant 0, R>0$ and $0 \leqslant\|u-v\| \leqslant R$. Assume $v$ is in $\operatorname{conv}\left(\operatorname{conv} S_{1} \backslash\left(\operatorname{conv} S_{2}\right)^{\circ}\right)$. Then we have

$$
\|u-v\|^{2}+r^{2} \leqslant R^{2}
$$

Proof: If $S_{1} \cap S_{2}$ is empty, the assertion is trivial. Let $S_{1} \cap S_{2}$ be not empty. Assume that $\|u-v\|^{2}+r^{2}>R^{2}$. Let $L:=\operatorname{conv}\left(S_{1} \cap S_{2}\right), l:=D(L, d)$ and $a:=l / 2$. Define $d:=\min \{\|x-u\|: x \in L\}$. Then we have $a^{2}+d^{2}=R^{2}$ and $a^{2}+(\|u-v\|-d)^{2}=r^{2}$. From this we get

$$
R^{2}-d^{2}=r^{2}-(\|u-v\|-d)^{2}
$$

and hence

$$
d=\frac{R^{2}-r^{2}+\|u-v\|^{2}}{2\|u-v\|}<\frac{\|u-v\|^{2}+\|u-v\|^{2}}{2\|u-v\|}=\|u-v\| .
$$

So the line which is determinated by $L$ separates conv $\left(\operatorname{conv} S_{1} \backslash\left(\operatorname{conv} S_{2}\right)^{\circ}\right)$ and $v$, which is a contradiction.

LEMmA 5. Define the following functions:

1. $f_{1}:[0,1 / 2] \longrightarrow \mathbb{R}, x \longmapsto 5 / 3-\left(2 \sqrt{3} \sqrt{1-x+x^{2}}+x\right) / 3$.

2. For $0 \leqslant w \leqslant 1 / 2: f_{2}:[0,1] \longrightarrow \mathbb{R}$,

$$
x \mapsto \frac{\left(1+x^{2}-w^{2}\right)(1+w)^{2}}{\left(x+\sqrt{(1+w)^{3}-w x^{2}}\right)^{2}} .
$$

3. For $w>0: f_{3}:(0,1] \longrightarrow \mathbf{R}, x \longmapsto\left(1+x^{2}-w^{2}\right) /\left(4 x^{2}\right)$.

4. $f_{4}:[0,1 / 2] \longrightarrow \mathbb{R}, x \longmapsto 1 / 4+\left(1+3 x-4 x^{2}\right) /(4(1+x))$.

Then we have:

1. $\max _{0 \leqslant x \leqslant 1 / 2} f_{1}(x)=f_{1}((1-\sqrt{3 / 11}) / 2)=(3-\sqrt{11 / 3}) / 2$.

2. $f_{2}^{\prime}(x) \begin{cases}<0 & \text { for } x<\left(1-w^{2}\right) /\left(\sqrt{1+6 w+w^{2}}\right) \\ =0 & \text { for } x=\left(1-w^{2}\right) /\left(\sqrt{1+6 w+w^{2}}\right) \\ >0 & \text { for } x>\left(1-w^{2}\right) /\left(\sqrt{1+6 w+w^{2}}\right)\end{cases}$

3. $f_{3}$ is monotonic decreasing.

4. $\max _{0 \leqslant x \leqslant 1 / 2} f_{4}(x)=f_{4}(\sqrt{3 / 2}-1)=3-\sqrt{6}$. 
The proof is straight forward.

Proof of Proposition 1: Without loss of generality, let $R=1$. From Lemma 4 we have

$$
\|u-v\|^{2}+r^{2} \leqslant 1
$$

and from Theorem 1 we have

$$
m\left(X, d^{2}\right)=\frac{1+r^{2}-\|u-v\|^{2}}{D\left(X, d^{2}\right)} .
$$

If $r=0$ we get $u=v$ and therefore $u$ is in $\operatorname{conv}\left(S_{1} \cap X\right)$. From this we get $D\left(X, d^{2}\right) \geqslant 3$ and hence

$$
m\left(X, d^{2}\right) \leqslant \frac{1}{3} .
$$

So assume $r>0$. Then it is easy to see that $\left|X \cap S_{1}\right|>1$.

CASE 1. $\left|X \cap S_{1}\right|=2$. So $X \cap S_{1}=\left\{y_{1}, y_{2}\right\}$ and $D\left(X, d^{2}\right) \geqslant\left\|y_{1}-y_{2}\right\|^{2}$. Let $g$ be the line with $v$ is in $g$, with $g$ perpendicular to the line segment $u v$ and let $\left\{x_{1}, x_{2}\right\}=S_{1} \cap g$. Then we have $\left\|x_{1}-x_{2}\right\|^{2}=4\left(1-\|u-v\|^{2}\right)$. Since $v$ is in $\operatorname{conv}\left\{y_{1}, y_{2}\right\}$ we get from Lemma $1\left\|y_{1}-y_{2}\right\| \geqslant\left\|x_{1}-x_{2}\right\|$ and therefore

$$
D\left(X, d^{2}\right) \geqslant 4\left(1-\|u-v\|^{2}\right) .
$$

Now we get with (1) and (2):

$$
m\left(X, d^{2}\right) \leqslant \frac{1+r^{2}-\|u-v\|^{2}}{4\left(1-\|u-v\|^{2}\right)} \leqslant \frac{2\left(1-\|u-v\|^{2}\right)}{4\left(1-\|u-v\|^{2}\right)}=\frac{1}{2} .
$$

CASE 2. $\left|X \cap S_{1}\right|>2$. From Lemma 3 we get points $y_{1}, y_{2}, y_{3}$ in $S_{1} \cap X$ with $v$ in conv $\left\{y_{1}, y_{2}, y_{3}\right\}$.

CASE 2.1. $u$ is not in conv $\left\{y_{1}, y_{2}, y_{3}\right\}$. Then there are two points in $\left\{y_{1}, y_{2}, y_{3}\right\}$, without loss of generality, $y_{1}$ and $y_{2}$, such that the line segment $y_{1} y_{2}$ does intersect the line segment $u v$. That is, $y_{1} y_{2} \cap u v=\{\bar{v}\}$. It follows that $\|u-\bar{v}\| \leqslant\|u-v\|$. Define two lines $g, h$ which are perpendicular to the line segment $u v$ with $\bar{v}$ on $g$ and $v$ on $h$. Let $\left\{y_{1}^{\prime}, y_{2}^{\prime}\right\}=S_{1} \cap g$ and $\left\{x_{1}, x_{2}\right\}=S_{1} \cap h$. From Lemma 1 we get $\left\|y_{1}^{\prime}-y_{2}^{\prime}\right\| \leqslant\left\|y_{1}-y_{2}\right\|$. Further we get

$$
\left(\frac{\left\|x_{1}-x_{2}\right\|}{2}\right)^{2}=1-\|u-v\|^{2} \leqslant 1-\|u-\bar{v}\|^{2}=\left(\frac{\left\|y_{1}^{\prime}-y_{2}^{\prime}\right\|}{2}\right)^{2}
$$

and hence

$$
D\left(X, d^{2}\right) \geqslant\left\|y_{1}-y_{2}\right\|^{2} \geqslant\left\|y_{1}^{\prime}-y_{2}^{\prime}\right\|^{2} \geqslant\left\|x_{1}-x_{2}\right\|^{2}=4\left(1-\|u-v\|^{2}\right) .
$$


Again we use (1) and (2) and get

$$
m\left(X, d^{2}\right) \leqslant \frac{1}{2}
$$

CASE 2.2. $u$ is in conv $\left\{y_{1}, y_{2}, y_{3}\right\}$. From Lemma 2 we have

$$
\max _{1 \leqslant i, j \leqslant 3}\left\|y_{i}-y_{j}\right\| \geqslant \sqrt{3}
$$

and so $D\left(X, d^{2}\right) \geqslant 3$. Assume $\|u-v\|>1 / 2$. Then we get together with (1) and (2)

$$
m\left(X, d^{2}\right) \leqslant \frac{2\left(1-\|u-v\|^{2}\right)}{3}<\frac{2(1-1 / 4)}{3}=\frac{1}{2} .
$$

So in the following we only have to consider the case $\|u-v\| \leqslant 1 / 2$.

We have $r$ in the interval $I=(0,1]$. Define the intervals

$$
\begin{gathered}
I_{1}:=\left(0, \sqrt{3}-\sqrt{1-\|u-v\|+\|u-v\|^{2}}\right] \\
I_{2}:=\left[\sqrt{3}-\sqrt{1-\|u-v\|+\|u-v\|^{2}}, \frac{1+\|u-v\|}{\sqrt{4\|u-v\|+1}}\right]
\end{gathered}
$$

and

$$
I_{3}:=\left[\frac{1+\|u-v\|}{\sqrt{4\|u-v\|+1}}, 1\right] .
$$

Therefore $r$ is in $I_{1} \cup I_{2} \cup I_{3}$.

CASE 2.2.1. $r$ is in $I_{1}$.

Since $D\left(X, d^{2}\right) \geqslant 3$ we get together with (2) and Lemma 5 ,

$$
\begin{aligned}
m\left(X, d^{2}\right) & \leqslant \frac{1+r^{2}-\|u-v\|^{2}}{3} \\
& \leqslant \frac{5}{3}-\frac{2 \sqrt{3} \sqrt{1-\|u-v\|+\|u-v\|^{2}}+\|u-v\|}{3} \\
& =f_{1}(\|u-v\|) \\
& \leqslant \frac{1}{2}\left(3-\sqrt{\frac{11}{3}}\right) \approx 0.5425728 .
\end{aligned}
$$

CASE 2.2.2. $r$ is in $I_{2}$. For $1 \leqslant i \leqslant 3$ define the lines $g_{i}, v+t\left(v-y_{i}\right)$ for $t \geqslant 0$. Since $y_{1}, y_{2}, y_{3}$ are points in $X$ and $X$ is connected there are at least two indices $i_{1}, i_{2} \in\{1,2,3\}$, $i_{1} \neq i_{2}$ and two points $a_{1}, a_{2}$ in $X$ with $a_{1} \in g_{i_{1}}$ and $a_{2} \in g_{i_{2}}$. Then define $x_{1}:=y_{i_{1}}$, $x_{2}:=y_{i_{2}}$ and $x_{3}:=y_{k}$, where $k \neq i_{1}, i_{2}$. From this it is clear that $\left\|x_{1}-a_{1}\right\| \geqslant\left\|x_{1}-v\right\|+r$ and $\left\|x_{2}-a_{2}\right\| \geqslant\left\|x_{2}-v\right\|+r$. So we have

$$
D\left(X, d^{2}\right) \geqslant \max \left\{\left\|x_{1}-x_{2}\right\|,\left\|x_{2}-x_{3}\right\|,\left\|x_{1}-x_{3}\right\|,\left\|x_{1}-v\right\|+r,\left\|x_{2}-v\right\|+r\right\}^{2} .
$$


If $\left\|x_{1}-x_{2}\right\|>2 \sqrt{1-\|u-v\|^{2}}$ we have $D\left(X, d^{2}\right)>4\left(1-\|u-v\|^{2}\right)$ and therefore together with (1) and (2), we have $m\left(X, d^{2}\right)<1 / 2$. So we only have to consider $\left\|x_{1}-x_{2}\right\| \leqslant$ $2 \sqrt{1-\|u-v\|^{2}}$.

Consider the arc joining $x_{1}$ and $x_{2}$ on $S_{1}$ which contains $x_{3}$. Let $x_{3}^{\prime}$ be on this arc with $\left\|x_{1}-x_{3}^{\prime}\right\|=\left\|x_{2}-x_{3}^{\prime}\right\|$. Then we have

$$
\max \left\{\left\|x_{1}-x_{3}\right\|,\left\|x_{2}-x_{3}\right\|\right\} \geqslant\left\|x_{1}-x_{3}^{\prime}\right\|
$$

and so we get

$$
D\left(X, d^{2}\right) \geqslant \max \left\{\left\|x_{1}-x_{2}\right\|,\left\|x_{1}-x_{3}^{\prime}\right\|,\left\|x_{1}-v\right\|+r,\left\|x_{2}-v\right\|+r\right\}^{2} .
$$

Now let $T: S_{1} \longrightarrow S_{1}$ be a rotation with centre $u$ and $T x_{3}^{\prime}=u+t(u-v)$ for a $t>0$. Then we have

$$
\left\|T x_{1}-v\right\|+r=\left\|T x_{2}-v\right\|+r .
$$

Of course $\left\|x_{1}-x_{2}\right\|=\left\|T x_{1}-T x_{2}\right\|,\left\|x_{1}-x_{3}^{\prime}\right\|=\left\|T x_{1}-T x_{3}^{\prime}\right\|$ and

$$
\max \left\{\left\|x_{1}-v\right\|+r,\left\|x_{2}-v\right\|+r\right\} \geqslant\left\|T x_{1}-v\right\|+r .
$$

So we get

$$
D\left(X, d^{2}\right) \geqslant \max \left\{\left\|T x_{1}-T x_{2}\right\|,\left\|T x_{1}-T x_{3}^{\prime}\right\|,\left\|T x_{1}-v\right\|+r\right\}^{2} .
$$

For short write again $x_{1}:=T x_{1}, x_{2}:=T x_{2}$ and $x_{3}:=T x_{3}^{\prime}$. Let $\bar{x}_{i}$ be the intersection point of the circle $S_{2}$ and the line segment $u x_{i}$, for $1 \leqslant i \leqslant 3$. Now define the following set $X^{\prime}: X^{\prime}$ is the arc joining $\bar{x}_{1}$ and $\bar{x}_{2}$ in $S_{2}$ with $u$ in the convex hull of this arc, together with the line segments $x_{i} \bar{x}_{i}$, for $1 \leqslant i \leqslant 3$. Then we have

1. $X^{\prime}$ is a compact, connected subset of conv $S_{1} \backslash\left(\operatorname{conv} S_{2}\right)^{\circ}$.

2. $u$ is in $\operatorname{conv}\left(S_{2} \cap X\right)$, and since $\left\|x_{1}-x_{2}\right\| \leqslant 2 \sqrt{1-\|u-v\|^{2}}$ also $v$ is in conv $\left\{x_{1}, x_{2}, x_{3}\right\}$.

3. $D\left(X^{\prime}, d^{2}\right)=\max \left\{\left\|x_{1}-x_{2}\right\|,\left\|x_{1}-x_{3}\right\|,\left\|x_{1}-v\right\|+r\right\}^{2} \leqslant D\left(X, d^{2}\right)$ and therefore $m\left(X^{\prime}, d^{2}\right) \geqslant m\left(X, d^{2}\right)$.

So in the following we only consider sets of the kind of $X^{\prime}$.

Let $z$ be the intersection point of $S_{1}$ and the line $u+t(u-v)$ for $t \geqslant 0$. If $S_{1} \cap S_{2}$ is not empty, let $y$ be in $S_{1} \cap S_{2}$. Otherwise let $y$ be the intersection point of $S_{1}$ and the line $u+t(u-v)$ for $t \leqslant 0$. Let $B$ be the shortest arc joining $y$ and $z$ on $S_{1}$. Let $g$ be the line which is perpendicular to $u v$ and which contains $v$ and define $p$ as the intersection point of $B$ and $g$. Each point $x$ on $B$ corresponds to an angle $\phi$ between the line segments $u v$ and $u x$. Therefore we write $x=x(\phi)$. Now define the angles $\phi_{1}$ and $\phi_{2}$ with $y=x\left(\phi_{1}\right)$ and $p=x\left(\phi_{2}\right)$. Clearly $\phi_{1} \geqslant 0$. Assume $\phi_{1}>\pi / 3$. Then we have 
$r>\sqrt{1-\|u-v\|+\|u-v\|^{2}} \geqslant(1+\|u-v\|) /(\sqrt{4\|u-v\|+1})$ and therefore $r$ is not in $I_{2}$. Hence we have $\phi_{1} \leqslant \pi / 3$. On the other hand we have $\cos \phi_{2}=\|u-v\|$. Since $0 \leqslant\|u-v\| \leqslant 1 / 2$ we get $\pi / 3 \leqslant \phi_{2} \leqslant \pi / 2$.

By definition of $X^{\prime}$ we have now $x_{3}=z, x_{1}=x(\phi)$ for $\phi_{1} \leqslant \phi \leqslant \phi_{2}$ and $x_{2}$ is the point on $S_{1}$ with $x_{1} \neq x_{2}$ and $\left\|x_{3}-x_{1}\right\|=\left\|x_{3}-x_{2}\right\|$.

Now we have

$$
\left\|x(\phi)-x_{3}\right\|=\sqrt{2} \sqrt{1+\cos \phi}
$$

and

$$
\|x(\phi)-v\|+r=\sqrt{1-2\|u-v\| \cos \phi+\|u-v\|^{2}}+r .
$$

It is easy to see that $\left\|x(\phi)-x_{3}\right\|$ is a monotonic decreasing function of $\phi$ and $\|x(\phi)-v\|+r$ is a monotonic increasing function of $\phi$. If $S_{1} \cap S_{2}$ is not empty we have $\left\|x\left(\phi_{1}\right)-v\right\|+r=2 r$. Since $\phi_{1}$ is the angle between the line segments uy, and uv we have

$$
r^{2}=1+\|u-v\|^{2}-2\|u-v\| \cos \phi_{1}
$$

and therefore

$$
\cos \phi_{1}=\frac{1+\|u-v\|^{2}-r^{2}}{2\|u-v\|}
$$

Hence we get

$$
\begin{aligned}
\left\|x\left(\phi_{1}\right)-x_{3}\right\| & =\sqrt{2} \sqrt{1+\cos \phi_{1}} \\
& =\sqrt{\frac{(1+\|u-v\|)^{2}-r^{2}}{\|u-v\|}} .
\end{aligned}
$$

Since $r$ is in $I_{2}$ we get

$$
\begin{aligned}
\left\|x\left(\phi_{1}\right)-x_{3}\right\|^{2} & =\frac{(1+\|u-v\|)^{2}-r^{2}}{\|u-v\|} \\
& \geqslant \frac{1}{\|u-v\|}\left[(1+\|u-v\|)^{2}-\frac{(1+\|u-v\|)^{2}}{4\|u-v\|+1}\right] \\
& =4 \frac{(1+\|u-v\|)^{2}}{4\|u-v\|+1} \\
& \geqslant 4 r^{2}
\end{aligned}
$$

and therefore

$$
\left\|x\left(\phi_{1}\right)-x_{3}\right\| \geqslant\left\|x\left(\phi_{1}\right)-v\right\|+r
$$


On the other hand we have $\left\|x\left(\phi_{2}\right)-x_{3}\right\| \leqslant\left\|x(\pi / 3)-x_{3}\right\|=\sqrt{3}$ and $\left\|x\left(\phi_{2}\right)-v\right\|+r \geqslant$ $\|x(\pi / 3)-v\|+r \geqslant \sqrt{3}$ since $r$ is in $I_{2}$. So there is $\phi_{0}$ in $\left[\phi_{1}, \phi_{2}\right]$ with

$$
\left\|x\left(\phi_{0}\right)-x_{3}\right\|=\left\|x\left(\phi_{0}\right)-v\right\|+r .
$$

If $S_{1} \cap S_{2}$ is empty we have $\phi_{1}=0$ and therefore we get $\left\|x\left(\phi_{2}\right)-x_{3}\right\| \leqslant \sqrt{3},\left\|x(0)-x_{3}\right\|=2$, $\left\|x\left(\phi_{2}\right)-v\right\|+r \geqslant \sqrt{3}$ and $\|x(0)-v\|+r \leqslant 1+r \leqslant 2$. As above there is $\phi_{0}$ in $\left[\phi_{1}, \phi_{2}\right]$ with

$$
\left\|x\left(\phi_{0}\right)-x_{3}\right\|=\left\|x\left(\phi_{0}\right)-v\right\|+r
$$

For short we define $w:=\|u-v\|$. Therefore we have

$$
1-2 w \cos \phi_{0}+w^{2}=2\left(1+\cos \phi_{0}\right)+r^{2}-2 \sqrt{2} r \sqrt{1+\cos \phi_{0}} .
$$

Since $\cos ^{2}\left(\phi_{0} / 2\right)=\left(1+\cos \phi_{0}\right) / 2$ and with $\psi:=\phi_{0} / 2$ we have

$$
1-2 w\left(2 \cos ^{2} \psi-1\right)+w^{2}=r^{2}+4 \cos ^{2} \psi-2 \sqrt{2} \sqrt{2} r \cos \psi .
$$

So we get the following equation for $\cos \psi$ :

$$
(4+4 w) \cos ^{2} \psi-4 r \cos \psi+r^{2}-(1+w)^{2}=0 .
$$

Solving this equation we get

$$
\cos \psi=\frac{r \pm \sqrt{(1+w)^{3}-w r^{2}}}{2(1+w)}
$$

Since $\sqrt{(1+w)^{3}-w r^{2}}>r$ we get

$$
\cos \psi=\frac{r+\sqrt{(1+w)^{3}-w r^{2}}}{2(1+w)}
$$

(Otherwise we have $\cos \psi<0$ and that is a contradiction to $0 \leqslant \psi \leqslant \pi / 4$.) Now we get together with (3)

$$
\begin{aligned}
D\left(X^{\prime}, d^{2}\right) & \geqslant\left(\sqrt{2} \sqrt{1+\cos \phi_{0}}\right)^{2} \\
& =4 \cos ^{2} \psi \\
& =\left[\frac{r+\sqrt{(1+w)^{3}-w r^{2}}}{(1+w)}\right]^{2}
\end{aligned}
$$

and hence

$$
m\left(X^{\prime}, d^{2}\right) \leqslant \frac{\left(1+r^{2}-w^{2}\right)(1+w)^{2}}{\left(r+\sqrt{(1+w)^{3}-w r^{2}}\right)^{2}}=f_{2}(r)
$$


From Lemma 5 we have

$$
\max _{x \in I_{2}} f_{2}(x)=\max \left\{f_{2}\left(\sqrt{3}-\sqrt{1-w+w^{2}}\right), f_{2}\left(\frac{1+w}{\sqrt{4 w+1}}\right)\right\}
$$

and

$$
f_{2}\left(\sqrt{3}-\sqrt{1-w+w^{2}}\right)=f_{1}(w) \leqslant \frac{1}{2}\left(3-\sqrt{\frac{11}{3}}\right) .
$$

The value $f_{2}((1+w) / \sqrt{4 w+1})$ will be calculated later.

CASE 2.2.3. $r$ is in $I_{3}$. We have $D\left(X, d^{2}\right) \geqslant 4 r^{2}$ and so

$$
m\left(X, d^{2}\right) \leqslant \frac{1+r^{2}-\|u-v\|^{2}}{4 r^{2}}=f_{3}(r)
$$

where $w$ is chosen as $\|u-v\|$. We have

$$
f_{3}\left(\frac{1+\|u-v\|}{\sqrt{4\|u-v\|+1}}\right)=f_{2}\left(\frac{1+\|u-v\|}{\sqrt{4\|u-v\|+1}}\right) .
$$

Since $f_{3}$ is a monotonic decreasing function on $I_{3}$ we have

$$
\begin{aligned}
f_{3}(r) & \leqslant f_{3}\left(\frac{1+\|u-v\|}{\sqrt{4\|u-v\|+1}}\right) \\
& =f_{4}(\|u-v\|) \\
& \leqslant f_{4}\left(\sqrt{\frac{3}{2}}-1\right) \\
& =3-\sqrt{6} .
\end{aligned}
$$

So we have

$$
m\left(X, d^{2}\right) \leqslant f_{3}(r) \leqslant 3-\sqrt{6}
$$

and we are done.

Proof of Theorem 2: Let $X$ be a compact, connected subset of $\mathbb{R}^{2}$. Then there is a circle $S_{1}$ with centre $u$ and radius $R$ and a circle $S_{2}$ with centre $v$ and radius $r$ with $X$ contained in conv $S_{1} \backslash\left(\operatorname{conv} S_{2}\right)^{\circ}$ and $u$ in $\operatorname{conv}\left(S_{2} \cap X\right)$ and $v$ in $\operatorname{conv}\left(S_{1} \cap X\right)$ (see Theorem 1). Therefore we get from Proposition 1

$$
m\left(X, d^{2}\right) \leqslant 3-\sqrt{6}
$$

and hence

$$
g_{2}\left(\mathbb{R}^{2}\right) \leqslant 3-\sqrt{6}
$$


Now we consider the set $A$ from Remark 1 in Section 2 . We have

$$
D\left(A, d^{2}\right)=\frac{6}{2 \sqrt{6}-3} .
$$

So we get with Wilson's Theorem,

$$
\begin{aligned}
M\left(A, d^{2}\right) & =\frac{1+\frac{3}{4 \sqrt{6}-6}-(\sqrt{3 / 2}-1)^{2}}{\frac{6}{2 \sqrt{6}-3}} \\
& =3-\sqrt{6} .
\end{aligned}
$$

\section{REFERENCES}

[1] J. Cleary, S.A. Morris and D. Yost, 'Numerical geometry - numbers for shapes', Amer. Math. Monthly 93 (1986), 117-127.

[2] O. Gross; 'The Rendezvous value of a metric space', in Advances in Game Theory, Ann. of Math. Studies 52 (Princeton University Press, Princeton, N.J., 1964), pp. 49-53.

[3] W. Stadje, 'A property of compact connected spaces', Arch. Math. (Basel) 36 (1981), 275-280.

[4] D.J. Wilson, 'A game with squared distance as payoff', Melbourne University Mathematics Research Report 1982, no. 16.

Institut für Mathematik

Universität Salzburg

Hellbrunnerstrasse 34

A-5020 Salzburg

Austria

e-mail: Friedrich.Pillichshammer@sbg.ac.at 